\title{
Perception paysanne et impact agro-pédologique du niveau de mécanisation agricole dans les zones cotonnières Centre et Ouest du Burkina Faso
}

\author{
Mathias Bouinzemwendé POUYA ${ }^{1 *}$, Moussa BONZI ${ }^{1}$, Zacharia GNANKAMBARY ${ }^{2}$, \\ Bazoumana KOULIBALY ${ }^{3}$, Idrissa OUEDRAOGO ${ }^{1}$, Jean Sibiri OUEDRAOGO ${ }^{4}$ et \\ Papaoba Michel SEDOGO ${ }^{4}$ \\ ${ }^{1}$ Institut de l'Environnement et de Recherches Agricoles (INERA).Centre de Recherches Environnementales, \\ Agricoles et de Formation de Kamboinsé. 01 B.P. 476 BF-Ouagadougou, Burkina Faso. \\ ${ }^{2}$ Bureau National des Sols (BUNASOLS). 03 B.P. 7142 BF-Ouagadougou 03, Burkina Faso. \\ ${ }^{3}$ Institut de l'Environnement et de Recherches Agricoles (INERA). Programme Coton. 01 BP 208. BF-Bobo- \\ Dioulasso 01 BF-Bobo-Dioulasso (Burkina Faso). Société Burkinabé des Fibres Textiles (SOFITEX). \\ ${ }^{4}$ Institut du Sahel. BP1580 Bamako, Mali. \\ *Auteur correspondant, E-mail : pouyabmathias@gmail.com
}

\section{RESUME}

Au Burkina Faso, la production cotonnière est assurée par un travail du sol à traction animale ou motorisée. Les sols assurant l'essentiel de cette production sont soit ferralitiques (à l'Ouest) soit ferrugineux tropicaux (au Centre). Pour évaluer l'impact socio-économique et agro-pédologique du type d'exploitation, des enquêtes (interviews semi-structurés et évaluation participative) ont été réalisées sur un échantillon de 315 exploitants et des mesures agro-pédologiques réalisées sur 140 champs dans les 2 zones cotonnières. Les résultats ont montré un faible niveau de mécanisation générale et trois groupes d'exploitants : les grands exploitants (type E1) qui disposent de grands moyens de productions, les exploitants moyens (type E2) et les petits exploitants (type E3) ayant de faibles revenus. Aussi, une proportionnalité existe entre les facteurs de production d'une exploitation et la catégorie socioprofessionnelle de l'exploitant. L'évaluation participative de l'impact des régimes de travail du sol sur les paramètres agronomiques et sur le sol a montré des perceptions divergentes en fonction de la zone de production. Sur les paramètres agronomiques les paysans des deux zones sont unanimes sur le fait que le travail profond du sol en traction animale ou motorisée permet d'améliorer les propriétés hydrodynamiques du sol et par conséquent entraine une bonne levée et une meilleure production des cultures. Quant à l'impact de la mécanisation agricole sur la fertilité du sol, les paysans de la zone Ouest à pluviosité > $900 \mathrm{~mm}$ et sur sols ferralitiques préconisent un travail minimum du sol. Tandis que ceux de la zone à pluviosité entre 700-900 $\mathrm{mm}$ et sur sols ferrugineux tropicaux recommandent le travail profond qui permettrait la récupération des terres encroûtés. Ces perceptions sont effectivement confirmées par les analyses agronomiques et pédoclimatiques faites dans les mêmes milieux. Pour une gestion durable des terres, il faut une intervention concertée avec les paysans. En outre les moyens de production doivent être vulgarisés en fonction des conditions pédoclimatiques de chaque zone.

(c) 2013 International Formulae Group. All rights reserved.

Mots clés: Impacts socio-économiques, mécanisation agricole, rendements, sol, zones cotonnières, Burkina Faso. 


\section{INTRODUCTION}

Les régions sahéliennes de l'Afrique de l'Ouest connaissent depuis des décennies, une pluviosité irrégulière et insuffisante. En outre les sols accusent une baisse du niveau de fertilité à cause de leur dégradation rapide après leur mise en culture ( Pallo et al., 2006 ; Sangaré et al., 2006). L'impact du travail du sol sur la conservation des eaux et des sols a fait l'objet de plusieurs travaux de recherche (Barro et al., 2005 ; Lahlou et al., 2005; Abdellaoui et al., 2010). Ces travaux ont montré l'intérêt du travail du sol dans l'amélioration des caractéristiques physiques (Zougmoré et al., 2006), entraînant très souvent une amélioration des rendements (Sawadogo et al., 2008). Dans le cas des zones cotonnières du Burkina Faso, la mécanisation agricole revêt une importance surtout pour la préparation du sol (Vall et al., 2006). La culture cotonnière a été le moteur de la mécanisation agricole par l'équipement des exploitations. Cependant des polémiques se lèvent autour de l'impact de la mécanisation agricole sur l'état structural du sol et sur les rendements des cultures (Lahlou et al., 2005). Le travail mécanisé du sol par la traction motorisée et attelée, entraine une dégradation plus accentuée qu'en culture manuelle et sous jachère. Les systèmes mécanisés créent une perturbation plus importante de la structure du sol, en relation avec la nature des équipements utilisés (Nouiri et al., 2004; Rhyan et al., 2008 ). Face à la méconnaissance de l'adaptabilité des chaînes d'outils proposées en fonction des conditions pédoclimatiques de la zone de production, le choix des techniques ou des équipements adaptés devient complexe pour les producteurs et les décideurs. Par ailleurs des travaux sur l'impact agronomique des techniques de travail du sol comme le labour et les sarclo-binages ont fait l'objet de thématiques de recherches (Barro et al., 2005 ; Son et al., 2004) . L'importance sociale et économique de la mécanisation agricole en zone cotonnière et l'impact de celle-ci sur la production du cotonnier et sur le sol jusque là peu exploré méritent d'être analysés de façon approfondie. Cette étude vise donc à générer des informations sur le poids socioéconomique de la mécanisation agricole au Burkina Faso. En outre elle vise à établir le rapport entre l'affectation des différents facteurs de production de chaque type d'exploitation et la capacité de celle-ci à gérer durablement la fertilité des champs.

\section{MATERIEL ET METHODES}

\section{Sites d'étude}

La présente étude s'est déroulée en milieu paysan dans les zones cotonnières Centre et Ouest du Burkina.

La zone cotonnière Centre couverte par la société FASOCOTON est dominée par un climat de type sub-sahélien au nord et au sud de type nord-soudanien (Guinko et Fontès, 1995). La pluviosité moyenne annuelle varie de 600 à $900 \mathrm{~mm}$. Les sols sont généralement de type ferrugineux tropicaux, peu profonds et peu fertiles, vulnérables à l'action de l'érosion et du ruissellement dans sa partie nord et centre. Dans sa partie sud, les sols sont assez fertiles de types sols peu évolués d'érosion et des vertisols (BUNASOLS, 1985).

La zone cotonnière Ouest ou zone SOFITEX est située entre les altitudes $9^{\circ} 30^{\prime}$ Sud et $14^{\circ} 00^{\prime}$ Nord. Cette zone est dans la zone climatique sub-soudanienne caractérisée par une moyenne pluviométrique annuelle variant entre 600 et $1200 \mathrm{~mm}$. Les sols sont à dominance des sols rouges faiblement ferralitiques. Ils sont pour la majorité apte à la production agricole (BUNASOLS, 1985) (Figure 1).

\section{Méthodologie}

L'étude a consisté d'une part à des enquêtes socio-économiques sur la mécanisation agricole et d'autre part, à une évaluation de son impact agronomique et pédologique dans les agro-systèmes cotonniers du Centre (FASOCOTON) et de l'Ouest (SOFITEX) du Burkina. Le choix des sites s'est fait de façon concertée avec les responsables des structures cotonnières. Les critères de choix des sites sont: la variabilité géographique de chaque zone et l'importance 
du site dans la production du coton. Pour le choix des producteurs, le principal critère est le type de mécanisation agricole. Le type de mécanisation agricole désigne tout matériel agricole utilisant les différentes formes d'énergie humaine: manuelle, animale et motorisée (FAO, 2008).

\section{Enquêtes socio-économiques}

La première enquête a consisté à des interviews semi-structurés (ISS) sur le niveau et l'importance socio-économique de la mécanisation agricole auprès de 315 exploitations cotonnières. La seconde enquête a consisté en une évaluation participative auprès de 135 exploitants dans la zone cotonnière du Centre (FASOCOTON), et de 180 paysans à l'Ouest dans la zone SOFITEX. Elle visait à déterminer la perception paysanne de l'impact de la mécanisation agricole sur la gestion de la fertilité et les composantes de rendements des cultures. Ce type d'enquête a utilisé la méthode de la notation matricielle adaptée de Lompo (2004).

\section{Principe de la méthode de notation matricielle}

La méthode de la notation matricielle est une méthode d'évaluation participative développée par Lompo (2004) pour évaluer la perception paysanne de l'impact d'innovations agricoles introduites. Elle a consisté dans cette étude, à discuter avec le producteur sur sa perception de l'impact des régimes de travail du sol sur les paramètres agronomiques. Des notes sont affectées par les producteurs à l'impact des différents régimes de travail du sol (grattage manuel, traction animale et traction motorisée) sur les composantes de rendements des cultures et sur le sol. Ces notes vont de 1 à 5 (1= effet le plus important à $5=$ effet le moins important).

La méthode de la notation matricielle passe par trois étapes pour avoir son caractère tangible :

- le classement empirique de l'impact du type de travail du sol sur les paramètres agropédologiques : à partir des notes recueillies auprès des producteurs enquêtés, on dresse un tableau de classement empirique de l'effet de chaque type de travail du sol.
- la distribution des fréquences d'importance de l'impact du type de travail du sol sur les paramètres agro-pédologiques : à l'issue des résultats du classement empirique, on établit un tableau de fréquence du nombre de fois qu'un type de travail du sol est classé dans un ordre de classement ou rang donné (1 à 5 selon la méthode);

- la distribution des probabilités cumulées de la perception de l'impact du type de travail du sol sur les paramètres agro-pédologiques : du précédent tableau, on calcule à partir de la formule ci-dessous la probabilité puis la probabilité cumulée d'un type de travail du sol d'être classée dans un ordre de classement rang (1 à 5 selon la méthode).

\section{$\mathrm{P}=\mathrm{F} /$ Nombre total des observations}

Avec $\mathrm{P}=$ Probabilité et $\mathrm{F}=$ Fréquence

Cette probabilité obtenue traduit la proportion de perception des producteurs visà-vis de l'effet du type de travail du sol sur les paramètres agronomiques et pédologiques. Enfin, on dresse à partir de ces probabilités cumulées, des courbes d'évolution des perceptions des producteurs de l'impact des trois types de travail du sol sur les composantes de rendements et sur l'état du sol suivant les rangs (1 à 5). Ces rangs sont des ordres de classement à partir desquels on a ou pas le maximum de perception. Généralement au rang 3, on enregistre le maximum de points de vue.

\section{Evaluations agronomiques et pédologiques}

Ces mesures agro-pédologiques ont concerné (Tableau 1) deux sites dans la zone cotonnière Ouest (SOFITEX) : Houet (Bala) et Tuy (Gombèlèdougou) et trois sites dans la zone Centre (FASOCOTON): Nahouri (Pô), Ganzourgou (Mogteedo) et Bam (Kongoussi). 140 producteurs qui pratiquent les mêmes modes de fertilisation et d'itinéraires techniques préconisés par les sociétés cotonnières ont été retenus parmi les 315 producteurs enquêtés. En outre, ils pratiquent soit le grattage manuel, soit la traction animale (ayant au moins une paire d'attelage) soit la culture motorisée (possédant au moins 
un tracteur). Ces mesures visaient à évaluer l'impact des trois régimes de travail du sol sur les composantes de rendements du cotonnier et sur les caractéristiques chimiques du sol.

Les mesures agronomiques ont porté sur les rendements coton-grain et sur la croissance du cotonnier. Ces mesures ont été effectuées dans cinq carrées de rendement de $9 \mathrm{~m}^{2}(3 \mathrm{~m} \times 3 \mathrm{~m})$ posées dans les champs de chaque producteur.

Des prélèvements de sol ont été effectués dans l'horizon de 0-20 cm à l'aide de la tarière pour une caractérisation chimique. Pour ce faire des échantillons moyens par carré de rendements ont été constitués à partir de cinq points de prélèvement dans chaque carré. Un échantillon représentatif par champ a été constitué du mélange des échantillons moyens des cinq carrés de rendements. On a 140 échantillons de sol au total. Les analyses ont porté sur l'azote total, le phosphore assimilable et total, le potassium total, le carbone organique et le $\mathrm{pH}$ eau. Elles ont été réalisées au laboratoire «Sol-Eau-Plantes» de l'Institut de l'Environnement et de Recherches Agricoles (INERA) de Kamboinsé. Le pH eau a été déterminé par la mesure potentiométrique dans un rapport sol/ eau de $1 / 2,5$. Le carbone total a été déterminé par la méthode Walkley-Black (1934). Pour la détermination des éléments totaux, les échantillons de sols ont été minéralisés avec une solution $\mathrm{H}_{2} \mathrm{SO}_{4}-\mathrm{Se}-\mathrm{C}_{7} \mathrm{H}_{6} \mathrm{O}_{3}$ en présence de $\mathrm{H}_{2} \mathrm{O}_{2}$. Puis $\mathrm{N}$ et $\mathrm{P}$ total dans la solution ont été déterminés en utilisant un colorimètre automatique (Skalar SANplus Segmented flow analyzer, Model4000-02, Breda, the Netherlands) et $\mathrm{K}$ a été déterminé au photomètre à flamme (Jencons PFP 7, Jenway LTD, Felsted, England). Le P assimilable a été déterminé selon la méthode BRAY I (Bray et Kurtz, 1946). Les logiciels GENSTAT 9è édition et Sphinx Lexica ont été utilisés pour l'analyse de variance des données et le test de Newman Kheuls pour la séparation des moyennes au seuil de probabilité de 5\%.

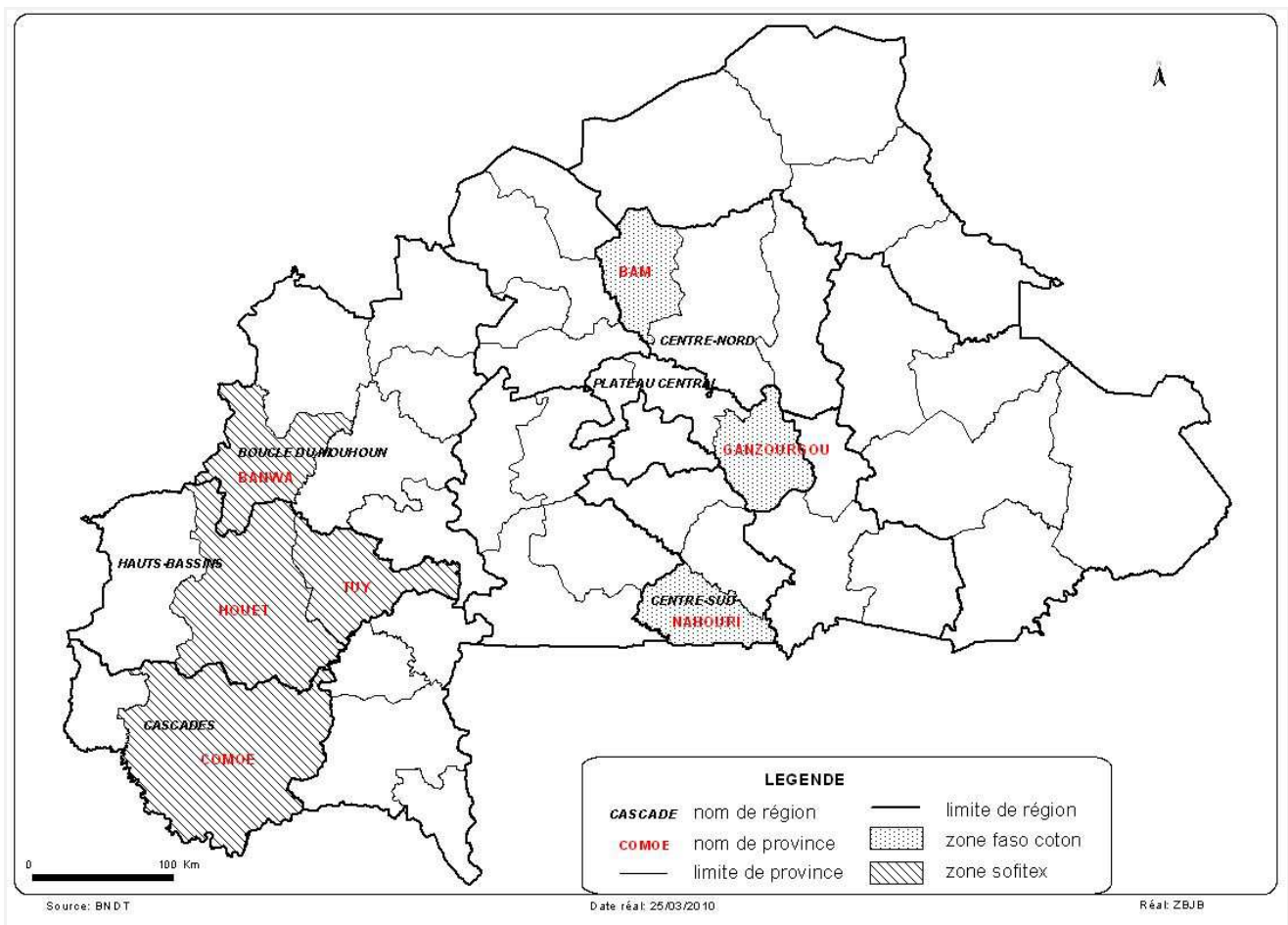

Figure 1 : Sites d'étude dans les zones cotonnière ouest et centre du Burkina Faso (Source BNDT, 2010). 
Tableau 1 : Effectif des exploitations suivant le type de mécanisation agricole.

\begin{tabular}{lcccccc}
\hline & \multicolumn{2}{c}{ Ouest (SOFITEX) } & \multicolumn{3}{c}{ Centre (FASO COTON) } \\
\cline { 2 - 7 } Type de mécanisation & Houet & Tuy & Nahouri & Gazourgou & Bam & Total \\
Exploitation manuelle & 15 & 15 & 10 & 10 & 10 & 60 \\
Exploitation attelée & 12 & 13 & 7 & 7 & 11 & 50 \\
Exploitation motorisée & 8 & 7 & 6 & 7 & 2 & 30 \\
Total & $\mathbf{3 5}$ & $\mathbf{3 5}$ & $\mathbf{2 3}$ & $\mathbf{2 4}$ & $\mathbf{2 3}$ & $\mathbf{1 4 0}$ \\
\hline
\end{tabular}

\section{RESULTATS}

Evaluation socio-économiques des exploitations cotonnières au Burkina Faso Caractéristiques socio-économiques des exploitations cotonnières

Le Tableau 2 présente les caractéristiques socio-économiques des exploitants cotonniers du Burkina Faso en fonction du niveau de mécanisation.

Les superficies cultivées sont plus importantes pour les exploitations motorisées (type E1) suivies de celles attelées (type E2) et manuelles (type E3). Les superficies emblavées par les exploitants mécanisés (type E1) sont trois fois plus grandes que celles des attelées (type E2). Les exploitations attelées (type E2) ont une superficie deux fois supérieure aux manuelles (type E3). En considérant les zones de production, les superficies emblavées de la zone cotonnière Ouest sont 2 à 4 fois supérieures à celles de la zone Centre. Elles sont de 7,86 ha pour la zone Ouest contre 3,37 ha pour la zone Centre en culture manuelle; 13,51 ha contre 5,02 ha en attelée et 44,25 ha contre 10,83 ha en culture motorisée.

La taille du troupeau croît en fonction $\mathrm{du}$ type d'exploitant. Le troupeau de l'exploitation est considéré dans cette étude en effectif de gros ruminants, et d'animaux de trait et de transport. Quelque soit le type d'animaux, les exploitations motorisées (type E1) et attelées (type E2) ont l'effectif le plus élevé.

En terme d'équipements agricoles, on trouve les outils aratoires pour le travail minimum ou superficiel du sol (dabas), les outils aratoires pour un travail peu profond $\mathrm{du}$ sol (charrues + buteurs) et ceux pour un travail profond du sol (tracteurs). L'importance de ces outils aratoires évolue en fonction des zones cotonnières. La zone cotonnière Ouest possède le plus grand nombre de ces trois types d'outils aratoires. Cette importance est aussi liée au type d'exploitant. Les exploitations motorisées (type E1) ont le plus grand nombre d'outils aratoires toutes catégories confondues.

Le revenu annuel de l'exploitation en zone cotonnière du Burkina Faso croît avec la catégorie de l'exploitant. Les revenus annuels des exploitants motorisés (type E1) vont de 1.933 .334 à 4.500 .000 francs CFA respectivement en zones cotonnières Centre et Ouest. Chez les exploitations attelées (type E2), les revenus sont de 521.296 francs CFA francs CFA et 652.157 francs CFA respectivement en zone Centre et Ouest.

Perception paysanne de l'impact de la mécanisation agricole sur les paramètres agronomiques et pédologiques

Perception paysanne de l'impact de la mécanisation agricole sur la croissance des cultures

Tous les producteurs interrogés (100\%) affirment que le travail du sol qu'il soit motorisé et attelé assure une meilleure croissance des cultures (Figures 2a et 2b).

Dans la zone cotonnière Ouest sur sols ferralitiques (Figure 2a) des taux d'acceptations de $97 \%$ et $100 \%$ sont reconnus aux cultures attelée et motorisée à l'ordre de classement 3. Au centre sur sols ferrugineux tropicaux (Figure 2b) déjà à l'ordre de classement 2 respectivement $100 \%$ et $58 \%$ des producteurs estiment que le travail du sol en motorisé et attelé permettent une bonne 
croissance des cultures. Quelques soient l'ordre de classement et la zone de production cotonnière, le grattage manuel est le moins apprécié par les producteurs sur la croissance des cultures.

Perception paysanne de l'impact de la mécanisation agricole sur les rendements des cultures

Les Figures $3 \mathrm{a}$ et $3 \mathrm{~b}$ présentent les perceptions paysannes de l'impact de la mécanisation agricole sur les rendements des cultures dans les zones cotonnières Ouest et Centre du Burkina Faso. Les paysans estiment que seuls les régimes de travail du sol en attelée et motorisée permettent d'avoir de meilleures productions. A l'Ouest sur sols ferralitiques $99 \%$ des paysans apprécient les régimes de travail du sol en attelée et en motorisée à l'ordre de classement 3 . Au centre sur sols ferrugineux tropicaux dans le même ordre de classement, la traction animale est plus appréciée par $97 \%$ des paysans, suivi de la motorisation par 96\%. Aussi le grattage manuel est le moins côté par les paysans.

Perception paysanne de l'impact de la mécanisation agricole sur le maintien de la fertilité des sols

Dans la zone Ouest sur sols ferralitiques (Figure 4a), les cultures manuelle et attelée sont perçues par les producteurs comme des pratiques moins dégradantes de la fertilité des sols avec des proportions d'acceptation chacune de $90 \%$. La motorisation est la moins cotée par les producteurs (39\%). Par contre dans la zone Centre sur sols ferrugineux (Figure 4b) les cultures attelées (89\%) et motorisée $(76 \%)$ sont considérées comme permettant un bon maintien de la fertilité à l'ordre de classement 3 .

Incidence de la mécanisation agricole sur la production du cotonnier et sur le sol en zones cotonnières du Burkina

Effet de régimes de travail du sol sur la production $d u$ cotonnier en zones cotonnières du Burkina

En zone cotonnière Ouest (Figures 5a et $5 \mathrm{~b}$ ), l'analyse de variance des paramètres de croissance et de rendement du cotonnier, révèle une différence significative au test de Newman Keulhs (seuil de 5\%). Entre la culture manuelle et celle motorisée, il existe une différence significative quelque soit le paramètre. La culture motorisée a permis d'avoir une meilleure croissance $(110 \mathrm{~cm})$ et le rendement en coton graine le plus élevé (2924 $\left.\mathrm{Kgha}^{-1}\right)$. Le grattage manuel a enregistré la croissance $(79 \mathrm{~cm})$ et le rendement coton-graine (1360 Kg.ha $\left.{ }^{-1}\right)$ les plus faibles.

En zone cotonnière Centre (Figures $6 \mathrm{a}$ et 6b) la croissance du cotonnier et le rendement coton-graine du cotonnier sont significativement influencés par les différents régimes de travail du sol. Les cultures attelée et motorisée ont un effet positif sur la croissance du cotonnier (respectivement 77 et $98 \mathrm{~cm}$ ). Les rendements en coton graine des exploitants manuels et motorisés sont les plus élevés respectivement $1304 \mathrm{Kg}$. ha ${ }^{-1}$ et 1567 Kg. ha ${ }^{-1}$.

Sur les sols ferralitiques de l'Ouest, les productions cotonnières sont plus importantes que sur les sols ferrugineux du Centre.

Effet de régimes de travail du sol sur les caractéristiques chimiques du sol en zones cotonnières du Burkina

Les différents régimes de travail du sol ont un effet sur les caractéristiques chimiques du sol (Tableau 4). D’une manière générale les teneurs en éléments nutritifs des sols sous ces différents régimes de travail du sol sont relativement élevées. En zone cotonnière Centre, le travail du sol en attelée et en motorisée ont un effet positif sur les différents paramètres chimiques du sol (matière organique, azote, potassium total, $\mathrm{C} / \mathrm{N} \mathrm{pH}$ ). Par contre en zone cotonnière Ouest, les champs ayant reçus le grattage manuel et la culture attelée ont les teneurs en éléments nutritifs les plus élevées. 
M. B. POUYA et al. / Int. J. Biol. Chem. Sci. 7(2): 489-506, 2013

Tableau 2 : Typologie des exploitants en zones cotonnières ouest et centre du Burkina Faso.

\begin{tabular}{|c|c|c|c|c|c|c|}
\hline \multirow{2}{*}{$\begin{array}{l}\text { Facteurs déterminants } \\
\text { le niveau de mécanisation }\end{array}$} & \multicolumn{3}{|c|}{$\begin{array}{c}\text { Zone cotonnière Ouest } \\
\text { (SOFITEX) } \\
\end{array}$} & \multicolumn{3}{|c|}{$\begin{array}{c}\text { Zone cotonnière Centre (FASO } \\
\text { COTON) }\end{array}$} \\
\hline & $\begin{array}{l}\text { Motorisé } \\
\text { (type E1) }\end{array}$ & $\begin{array}{l}\text { Attelé } \\
\text { (type E2) }\end{array}$ & $\begin{array}{l}\text { Manuel } \\
\text { (type E3) }\end{array}$ & $\begin{array}{l}\text { Motorisé } \\
\text { (type E1) }\end{array}$ & $\begin{array}{l}\text { Attelé } \\
\text { (typeE2) }\end{array}$ & $\begin{array}{l}\text { Manuel } \\
\text { (type E3) }\end{array}$ \\
\hline \multicolumn{7}{|l|}{ Caractéristiques générales de l'exploitation } \\
\hline Durée de mise en culture des champs (année) & 47 & 30 & 29 & 25 & 20 & 30 \\
\hline Superficies totales emblavées (ha) & 44 & 14 & 8 & 11 & 5 & 3 \\
\hline Superficie des champs de coton (ha) & 18 & 5 & 3 & 5 & 3 & 2 \\
\hline Nombre d'actifs & 22 & 8 & 4 & 12 & 5 & 5 \\
\hline \multicolumn{7}{|l|}{ Biens vivants de l'exploitation } \\
\hline Gros ruminants & 37 & 6 & 0 & 15 & 6 & 2 \\
\hline Animaux de trait + transport (bœufs+ ânes) & 10 & 4 & 0 & 8 & 4 & 1 \\
\hline Nombre moyen d'animaux /exploitation & 47 & 10 & 0 & 23 & 10 & 3 \\
\hline \multicolumn{7}{|l|}{ Nombre d'équipements agricoles de l'exploitation } \\
\hline Outils aratoires à travail minimum du sol* & 28 & 9 & 4 & 12 & 9 & 7 \\
\hline Outils aratoires à travail peu profond du sol* & 9 & 3 & 0 & 4 & 3 & 0 \\
\hline Outils aratoires à travail profond du sol* & 1 & 0 & 0 & 1 & 0 & 0 \\
\hline Nombre moyen d'outils aratoires/exploitation & 38 & 12 & 4 & 17 & 12 & 7 \\
\hline Semoirs & 2 & 1 & 0 & 1 & 1 & 0 \\
\hline Charrettes & 3 & 1 & 0 & 1 & 1 & 1 \\
\hline Appareils phytosanitaires & 3 & 1 & 1 & 1 & 1 & 1 \\
\hline Revenu annuel de l'exploitation (FCFA) & 4500000 & 652157 & 241071 & 1933334 & 521296 & 289.375 \\
\hline
\end{tabular}

outils à travail minimum du sol*= dabas et assimilées ; outils à travail peu profond du sol*= houes mangas, charrues, butteurs ;

outils à travail profond du sol*= tracteurs et assimilés. 
M. B. POUYA et al. / Int. J. Biol. Chem. Sci. 7(2): 489-506, 2013

Tableau 3 : Régimes de travail du sol sur les caractéristiques chimiques du sol.

\begin{tabular}{|c|c|c|c|c|c|c|c|c|}
\hline \multirow{2}{*}{ Zone } & \multirow{2}{*}{$\begin{array}{l}\text { Travail } \\
\text { du sol }\end{array}$} & \multirow{2}{*}{$\begin{array}{c}\mathrm{Nt} \\
\text { g.kg-1 }\end{array}$} & \multirow[t]{2}{*}{ Pass } & \multirow{2}{*}{$\begin{array}{c}\text { Pt } \\
\text { mg.kg }^{-1}\end{array}$} & \multirow[t]{2}{*}{$\mathbf{K}$} & \multirow{2}{*}{$\mathbf{C} / \mathbf{N}$} & \multirow{2}{*}{$\operatorname{MO}(\%)$} & \multirow{2}{*}{ pH KCl } \\
\hline & & & & & & & & \\
\hline \multirow{4}{*}{ Centre } & Manuel & $0,52 \pm 0,03$ & $3,5 \pm 1,2$ & $255,1 \pm 45,1$ & $518,5 \pm 35,5$ & $12,8 \pm 2,6 \mathrm{a}$ & $1,2 \pm 0,22$ & $5,2 \pm 0,4$ \\
\hline & Attelé & $0,57 \pm 0,03$ & $4,0 \pm 0,7$ & $226,3 \pm 24,2$ & $676,2 \pm 77,2$ & $14,9 \pm 0,5 \mathrm{ab}$ & $1,4 \pm 0,01$ & $5,6 \pm 0,1$ \\
\hline & Motorisé & $0,51 \pm 0,02$ & $6,2 \pm 1,5$ & $223,5 \pm 22,5$ & $963,1 \pm 10,1$ & $17,3 \pm 1,9 \mathrm{c}$ & $1,5 \pm 0,06$ & $5,6 \pm 0,2$ \\
\hline & Fpr. & 0,723 & 0,252 & 0,177 & 0,479 & 0,024 & 0,634 & 0,06 \\
\hline \multirow{4}{*}{ Ouest } & Manuel & $0,62 \pm 0,1$ & $9,81 \pm 0,3$ & $211,1 \pm 20,11$ & $987,1 \pm 44,1$ & $15,6 \pm 0,4$ & $1,8 \pm 0,3$ & $5,9 \pm 0,4$ \\
\hline & Attelé & $0,56 \pm 0,04$ & $10,4 \pm 0,21$ & $168,1 \pm 23,13$ & $979,1 \pm 33,1$ & $15,5 \pm 0,3$ & $1,4 \pm 0,1$ & $5,6 \pm 0,1$ \\
\hline & Motorisé & $0,38 \pm 0,17$ & $10,3 \pm 0,1$ & $222,3 \pm 30,3$ & $639,3 \pm 104,3$ & $14,8 \pm 0,4$ & $0,9 \pm 0,4$ & $5,6 \pm 0,4$ \\
\hline & Fpr. & 0,483 & 0,981 & 0,767 & 0,832 & 0,809 & 0,339 & 0,18 \\
\hline
\end{tabular}




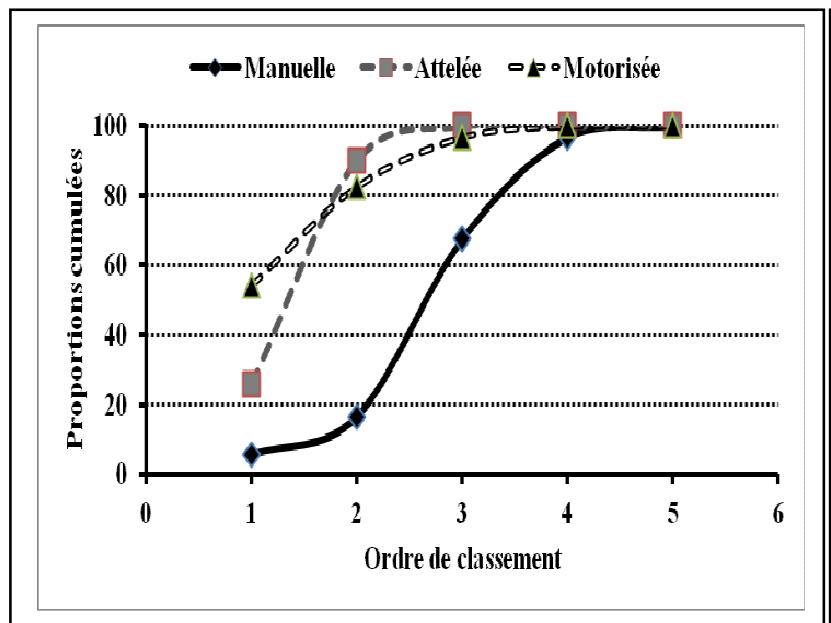

Figure 2a

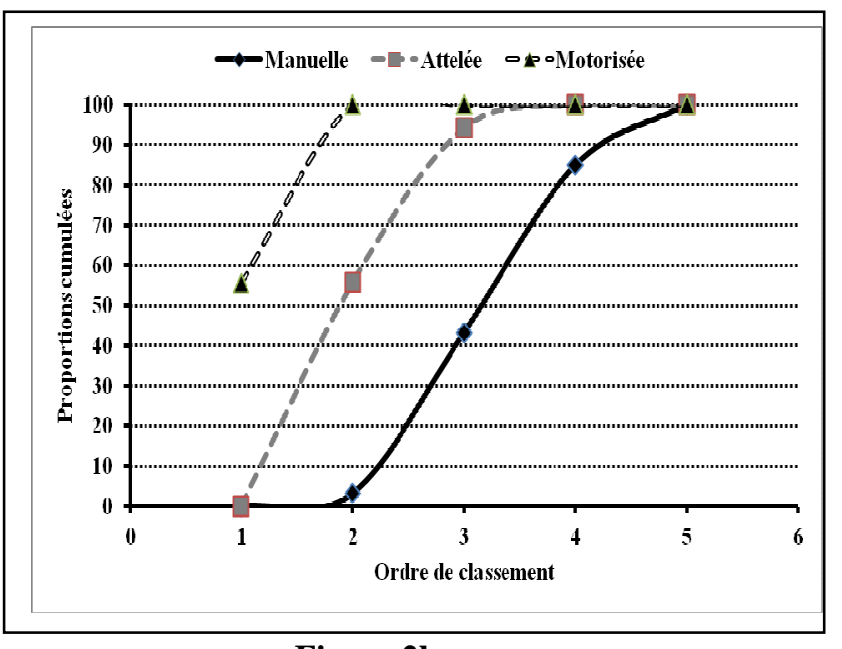

Figure 2b

Figure 2 : Perception paysanne de l'impact de la mécanisation agricole sur la levée des cultures en zones cotonnières Ouest (a) et Centre (b) du Burkina Faso. 


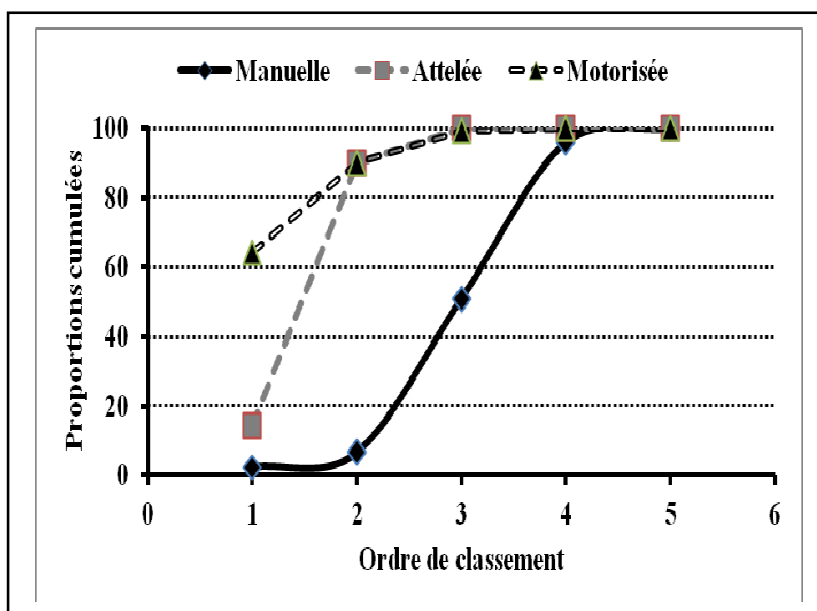

Figure 3a

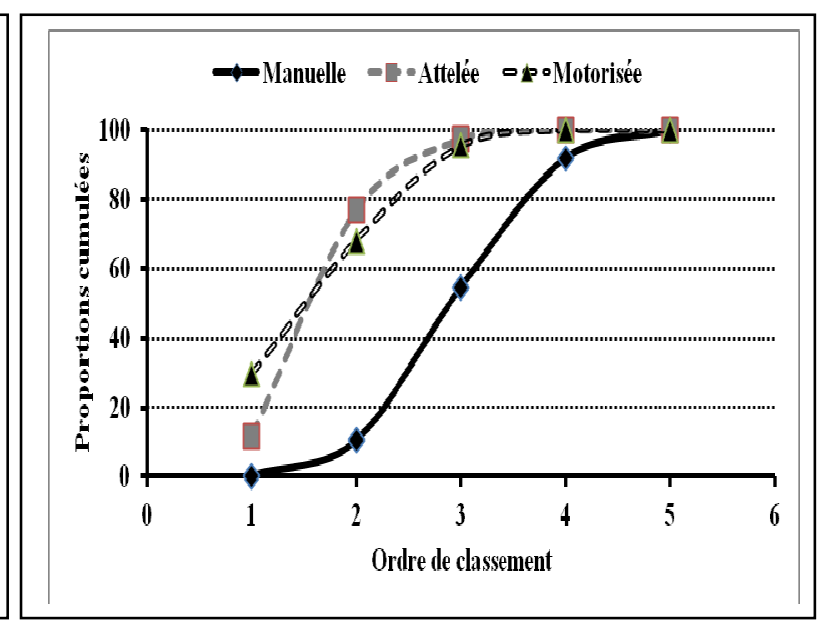

Figure 3b

Figure 3: Perception paysanne de l'impact de la mécanisation agricole sur les rendements des cultures en zones cotonnières Ouest (a) et Centre (b) du Burkina Faso. 


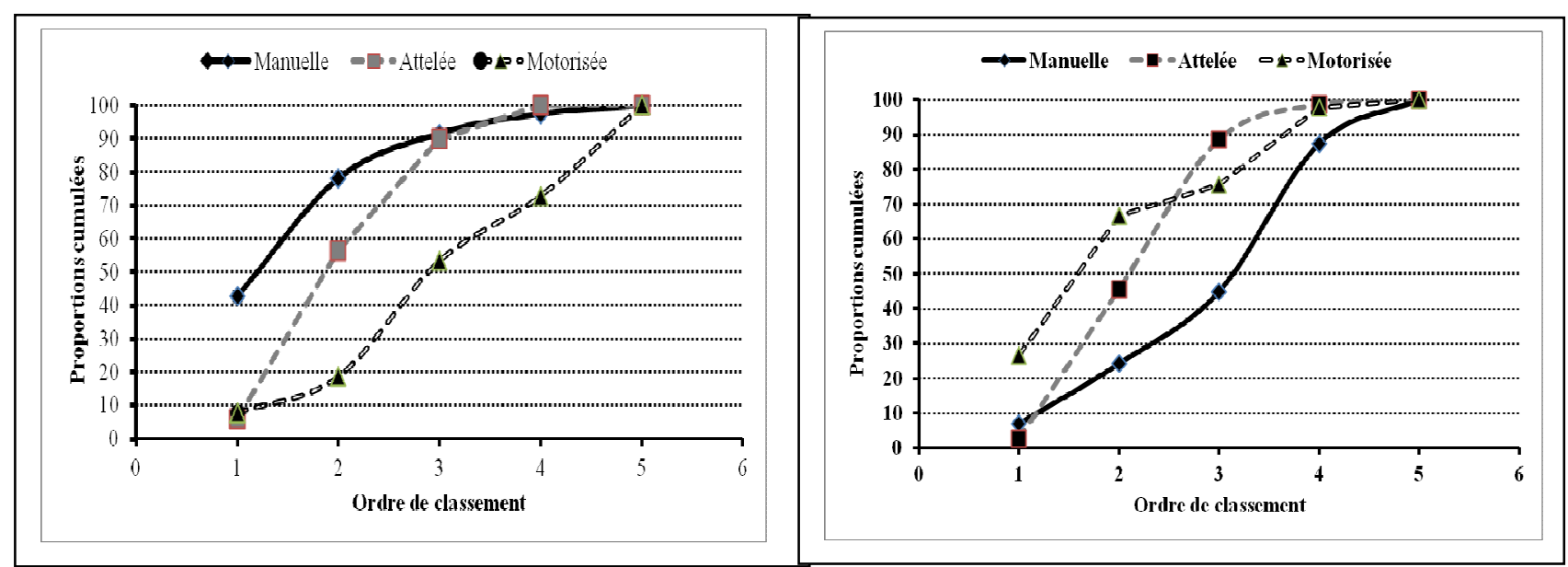

Figure 4a : Zone cotonnière Ouest

Figure 4b: Zone cotonnière Centre

Figure4 : Courbe d'évolution de la perception paysanne de l'impact de la mécanisation agricole sur la dégradation des sols en zones cotonnières Ouest (a) et Centre (b) du Burkina Faso. 
M. B. POUYA et al. / Int. J. Biol. Chem. Sci. 7(2): 489-506, 2013

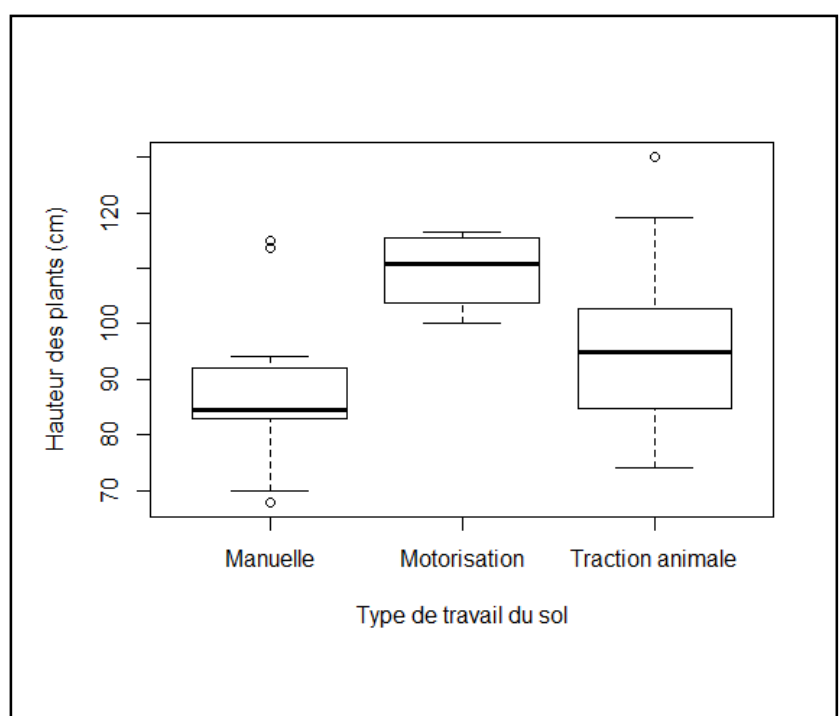

Figure 5 a $(F>P r .=0,027 ; S)$

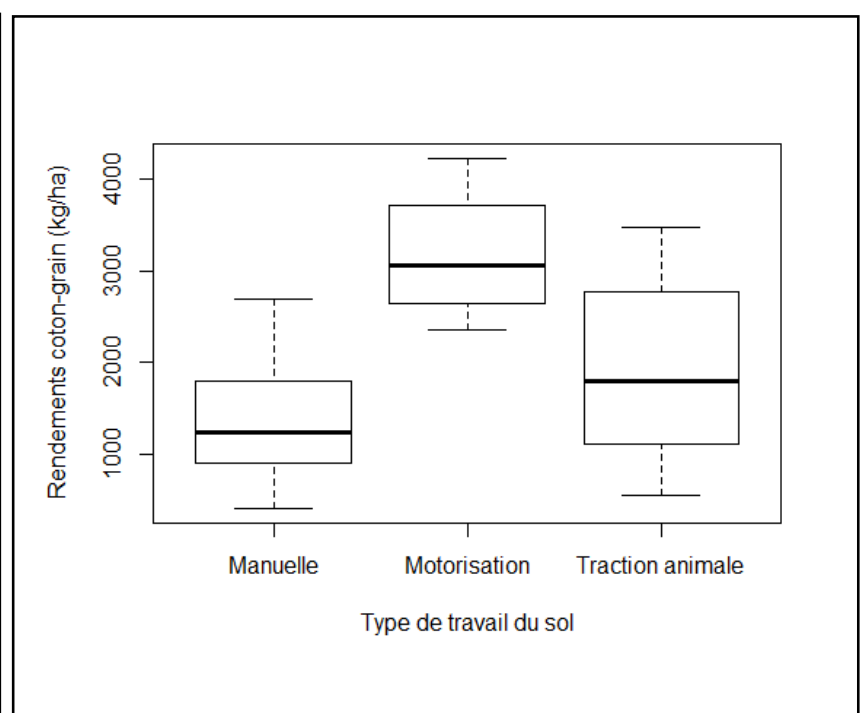

Figure 5b $(F>P r .=0,009 ; S)$

Figure 5 : Régimes de travail du sol sur la croissance (a) et le rendement du cotonnier (b) en zone cotonnière Ouest du Burkina Faso. 


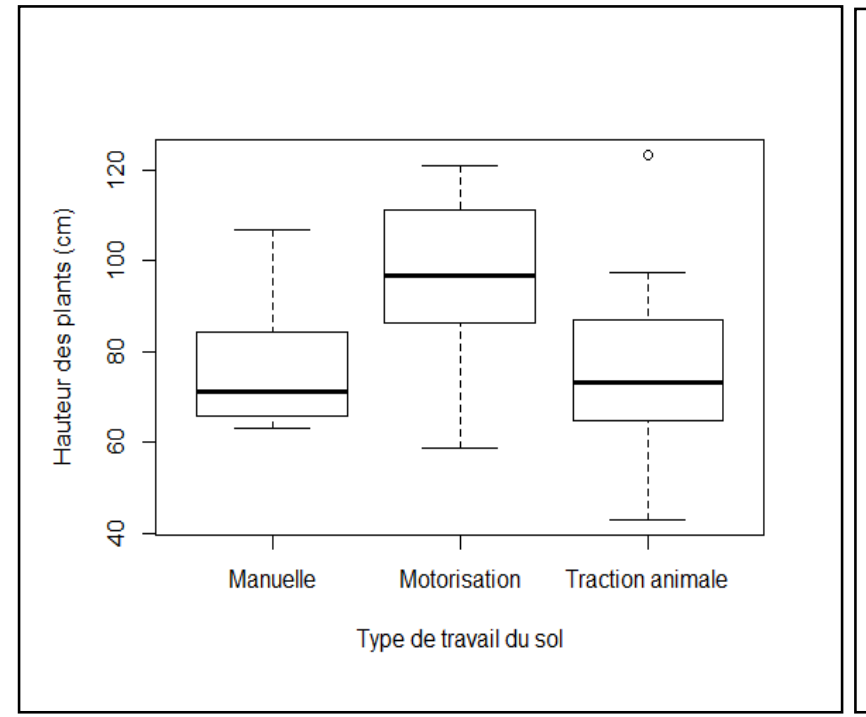

Figure 6a $(F>P r .=0,019 ; S)$

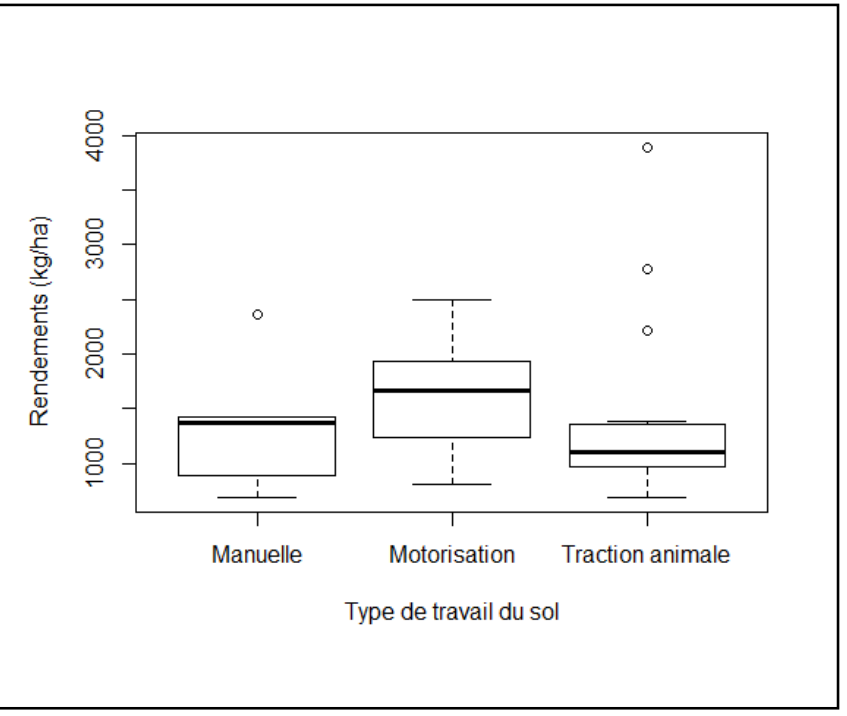

Figure 6b $(F>P r .=0,476 ; S)$

Figure 6: Régimes de travail du sol sur la croissance (a) et le rendement du cotonnier (b) en zone cotonnizaère Centre du Burkina Faso. 


\section{DISCUSSION}

Caractéristiques générales de l'exploitation en zones cotonnières

Une typologie se dégage en relation étroite avec le type de mécanisation agricole. Le premier type E1 possède de grandes superficies, une importante force de travail, un effectif élevé d'animaux, d'importants moyens de production et des revenus agricoles assez élevés. Le second type E2 à la différence du premier ne dispose pas d'engin motorisé. En outre les facteurs de production sont en nombre réduit. Le troisième type E3 se distingue des autres par un faible moyen de production et connait des difficultés pour réaliser des investissements. Aussi, il possède des équipements agricoles rudimentaires pour la préparation des champs. L'établissement d'une typologie des exploitants cotonniers permet de savoir d'emblée les pratiques agricoles adoptées par chaque type d'exploitant. Aussi il permet en fonction du mode d'affectation des facteurs de productions d'évaluer le potentiel de l'exploitation à gérer la fertilité des sols. Cette typologie s'oriente sur le type de mécanisation agricole. Elle présente l'avantage d'être exhaustive, en ce sens qu'elle prend en compte des déterminants comme la taille du cheptel, le nombre d'actifs par exploitation, le nombre d'équipements et le revenu agricole qui permettent de statuer sur le potentiel, voire l'efficience des exploitations cotonnières à gérer durablement la fertilité de leurs champs. En effet dans les exploitations de taille moyenne et grande, propriétaires d'un cheptel important et cultivant de grandes surfaces, le carbone initialement disponible est contenu dans les déjections animales et, dans une moindre mesure, dans les biomasses végétales produites. Selon Dugué et al. (2004), Faure et al. (2010), la quantité de carbone initialement disponible est liée à la taille du cheptel, à la surface totale cultivée, et à la part de l'assolement en céréales sèches (sorgho, mil) qui produisent une grande quantité de biomasse (4 t de MS/ha contre $1 \mathrm{t}$ de MS en moyenne pour le coton).
Sous un autre angle l'importance socio-économique des exploitations revêt une situation de cause à effet. De fait une proportionnalité existe entre le niveau de mécanisation agricole, les superficies emblavées, le nombre d'actif, les équipements agricoles et le revenu agricole. Plus les exploitations agricoles ont un niveau élevé de mécanisation, plus les superficies exploitées sont importantes. La mécanisation agricole constitue une stratégie d'occupation de l'espace et d'appropriation des terres (FAO, 2008).

Le nombre d'actif par exploitation agricole croît avec le niveau de mécanisation agricole. La mécanisation ne tend pas à substituer la main d'œuvre familiale. La disponibilité d'une force de travail plus abondante au sein des exploitations motorisées, explique leur tendance à accroître leurs superficies.

L'acquisition d'équipements agricoles est une phase stratégique pour l'exploitation. Elle constitue un facteur essentiel pour l'accroissement rapide des superficies (Barro et al., 2005) et l'intensification de la production (Raubec, 2001). En général, quelque soit le type d'équipements, le nombre croît des exploitations manuelles à celles motorisées.

L'adoption de la motorisation agricole renforce aussi la composante élevage de l'exploitation surtout avec la traction animale. De manière systématique toutes les exploitations possèdent des animaux aussi bien pour les opérations culturales, pour l'élevage que pour le fumier. Cela s'inscrit dans un processus d'intégration agricultureélevage au Burkina-Faso. Pour Vall et al. (2006), l'intégration agriculture-élevage correspond à une forme d'intensification basée sur une gestion raisonnée des flux de matière organique et d'énergie disponibles sur les terroirs et les exploitations. A l'échelle de l'exploitation, l'attelage est un maillon essentiel de l'intégration par son apport d'énergie agricole et pour le recyclage des résidus de culture (Mbetid-Bessane et al., 2010). Une des caractéristiques clés des 
exploitations agricoles est le revenu agricole qui détermine la capacité de celles-ci à la modernisation à travers l'acquisition des facteurs de productions (équipements et intrants agricoles). Le revenu de l'exploitation provient non seulement des récoltes mais aussi des prestations de services extraexploitation. Pour Faure et al. (2010), le revenu monétaire agricole s'accroît fortement avec le niveau de mécanisation, justifiant l'engouement pour la traction animale ou la motorisation et les intrants agricoles.

Perception paysanne de l'impact de la mécanisation sur la production agricole et sur le sol

On observe une convergence des perceptions paysannes de l'impact de la mécanisation agricole sur la croissance et les rendements des cultures en zones cotonnières du Burkina. Les cultures motorisée et attelée sont perçues comme celles qui entrainent une bonne levée et une meilleure production des cultures. L'action des outils aratoires sur la croissance et les rendements des cultures a été étudiée par Bessam et al. (2001) ; Lahlou et al. (2005) ; Abdellaoui et al. (2010). L'effet du type de travail du sol sur la levée des plantes dans les deux zones cotonnières se justifie par le fait que le travail par les engins mécanisés est plus profond et ameubli le sol auparavant compact. Ce qui permet une bonne infiltration et une meilleure rétention d'eau du sol. Comme le souligne Son et al. (2004) l'utilisation du décompacteur du sol à l'état sec contribue à l'amélioration de l'humidification du sol. Le grattage manuel n'apporte pas de modifications profondes au profil cultural, mais lorsqu'il est assez profond, il améliore dès les premières pluies les propriétés hydrodynamiques du sol telles que l'infiltration (Kribaa et al., 2001 ; Mando et al., 2005). Aussi l'amélioration des techniques de travail du sol a comme résultat, une augmentation des rendements des cultures (Nouiri et al., 2004).

Cependant les avis divergent au sujet des perceptions paysannes de l'impact de la mécanisation sur le maintien de la fertilité des sols. Ce qui s'explique par la différence des réalités pédologiques et climatiques de ces deux zones. En effet dans la zone cotonnière du Centre, les sols sont naturellement peu fertiles et dégradés (zippelé, croûtes dénudées); les pluies sont généralement insuffisantes pour faciliter le travail du sol. Barro et al. (2005) ont montré que dans les régions Centre et Nord du Burkina Faso, les superficies des sols dégradés et dénudés (zippélé) sont considérables (plus de $24 \%$ de la surface agricole totale). D'où la nécessité d'engins motorisés pour pratiquer les techniques de travail et de récupération des sols dégradés telles que les zaï, les demilunes. Dans la zone cotonnière Ouest, les producteurs estiment que le travail réduit du sol (grattage manuel, traction animale) permet le maintien de la fertilité des sols. En effet, les sols de cette zone sont encore fertiles, le grattage manuel se limitant à un travail superficiel du sol sans perturbation de la structure serait mieux adapté. Par contre un travail profond du sol avec le tracteur désorganiserait la structure du sol en faisant en même temps remonter la terre infertile en surface (Barro et al., 2009 ; Neto et al., 2010). Aussi la zone Ouest est très pluvieuse, par conséquent le travail du sol en traction motorisée ou attelée entraîne une dégradation du sol par l'érosion hydrique. Par ailleurs, l'impact des pluies sur les sols explique les perceptions paysannes. La zone cotonnière Ouest enregistre une pluviosité abondante. Un travail profond du sol entrainerait le décapage de la couche arable. Par contre, dans la zone Centre où la pluviosité est relativement faible, un travail profond du sol renforcé par des ouvrages antiérosifs (zaï, demi-lune...) permet une meilleure rétention de l'eau et par conséquent une bonne gestion de l'eau au niveau de la parcelle.

\section{Effet des régimes de travail du sol sur la production cotonnière et sur le sol}

L'adoption d'un nouveau mode de travail du sol a des effets sur les composantes de rendements des cultures et sur le sol. L'effet du type de travail du sol sur le 
rendement en coton graine est positif avec les cultures attelées et motorisées au Centre. Le travail profond du sol convient vraisemblablement à la zone cotonnière Centre. En zone cotonnière Ouest le travail minimum préserve mieux les propriétés chimiques. Cette différence serait imputable à la position agro-climatique de chaque zone qui détermine les conditions de mise en place des cultures dont la précarité s'accentue des zones plus arrosées vers les zones moins arrosées. Abdellaoui et al. (2010) ont obtenu en Algérie sous culture de céréales, une amélioration de rendements et des propriétés physico-chimiques du sol avec le travail réduit $\mathrm{du}$ sol. Les valeurs du $\mathrm{pH}$ montrent que les sols sont en général acides. Dans la zone Centre cette acidité est beaucoup plus importante dans les sols en culture manuelle. Ce qui pourrait augmenter à long terme le risque de toxicité aluminique pour le cotonnier. Pour Di Vecchia et al. (2001), la valeur $\mathrm{pH}$ eau égale à 5,5 est un seuil majeur pour le cotonnier en dessous de laquelle la présence d'aluminium échangeable entraîne des risques de toxicité aluminique pour le cotonnier. Les teneurs en matière organique du sol (MOS) plus élevées sous exploitation motorisée $(1,5 \%)$ et attelée $(1,4 \%)$ peuvent être attribuées à l'utilisation de la matière organique en quantité élevée dans cette zone.

Les teneurs en azote, en phosphore assimilable et en potassium sont plus élevées sous exploitations attelée $(0,57 \mathrm{~g} / \mathrm{Kg} \mathrm{N} ; 4,0$ $\mathrm{mg} / \mathrm{Kg} \mathrm{P} ; 676,2 \mathrm{mg} / \mathrm{Kg} \mathrm{K})$ et motorisée $(0,51 \mathrm{~g} / \mathrm{Kg} \mathrm{N} ; 6,2 \mathrm{mg} / \mathrm{Kg} \mathrm{P} ; 963,1 \mathrm{mg} / \mathrm{Kg}$ K). Zougmoré et al. (2006) ont évalué dans cette zone (Centre-nord) $24 \%$ de sols très dégradés et dénudés sous formes de cuirasses. De ce fait, Barro et al. (2005) ont recommandé l'usage des techniques de CES/DRS et le travail profond du sol pour décompacter les croûtes afin d'améliorer les propriétés physico-chimiques de ces sols et ainsi libérer les nutriments. Dans la zone Ouest les valeurs de $\mathrm{pH}$ eau, les teneurs en MOS, en azote, en potassium sont plus élevées sous cultures manuelles ( $\mathrm{pHeau}=$ 5,9 ; 1,8\% MOS, 0,62 g/Kg N ; 987,1 mg/Kg) suivie de celle attelée ( $\mathrm{pHeau}=5,6 ; 1,4 \%$ MOS, $0,56 \mathrm{~g} / \mathrm{Kg} \mathrm{N}$; 979,1 mg/Kg). Ces résultats dans les deux zones corroborent les perceptions paysannes de l'impact de la mécanisation agricole sur le sol et sur la production du coton. Le régime de travail du sol doit être réorienté en fonction des conditions pédologiques-climatiques de chaque agro-système cotonnier.

\section{Conclusion}

Cette étude a servi de base comparative entre deux zones cotonnières contrastées du point de vue agro-pédoclimatique et socio-économique en termes de données sur l'importance socio-économique de la mécanisation agricole et des changements induits par celles-ci sur la production du coton et sur le sol. Après 30 années de développement dans les zones cotonnières du Burkina Faso, la motorisation agricole occupe une place centrale dans les stratégies individuelles et collectives des producteurs. Cependant, le niveau de mécanisation agricole dans les zones cotonnières demeure faible. Il ressort de cette étude trois catégories d'exploitants corrélées étroitement avec le type de mécanisation agricole. Une relation existe entre les différents facteurs de productions (les superficies emblavées; la main d'œuvre, la taille $\mathrm{du}$ troupeau et les équipements agricoles). Cette relation renforce les efforts vers l'intensification de l'exploitation. Cependant il n'existe pas de rapport étroit entre la catégorie de l'exploitant et sa capacité à gérer durablement la fertilité des sols. Dans la zone cotonnière Centre les exploitants moyens et grands possédant beaucoup de facteurs de production gèrent mieux la fertilité de leur champ comparativement aux petits exploitants. C'est l'inverse en zone cotonnière Ouest (sols assez fertiles) où les petits exploitants menant un travail minimum du sol préservent mieux la fertilité du sol. La capacité à bien gérer le capital productif est surtout imputable aux conditions pédoclimatiques de la zone de production. L'étude suggère de ce fait, de réorienter les 
modes d'affectation des facteurs de producteurs en fonction du climat et des zones agro-écologiques des zones de production cotonnière.

\section{REFERENCES}

Abdellaoui Z, Teskrat H, Zaghouane ABO. 2010. Étude comparative de l'effet du travail conventionnel, semis direct et travail minimum sur le comportement $d$, une culture de blé dur dans la zone subhumide. Options Méditerranéennes, 96: 71-87.

Barro A, Zougmoré R, Taonda JB. 2005. Mécanisation de la technique du zaï manuel en zone semi-aride. Cahiers Agriculture, 14(6): 549-559.

Barro A, Zougmoré R, Sédogo MP. 2009. Evaluation de la faisabilité de trois types de travail du sol : application du modèle Sarra dans le Plateau central au Burkina Faso. Sécheresse, 20(4): 338-345.

Bessam F, Mrabet R. 2001. Time influence of no tillage on organic matter and its quality of a vertic Calcixeroll in a semiarid area of morocco. In Proceedings of international congress on conservation agriculture, 1-5 October 2001, Madrid (Spain), 281-286.

Bray RH, Kurtz LT. 1945. Determination of total, organic, and available forms of phosphorus in soils. Soil Science, 59: 3945.

BUNASOLS. 1985. État de connaissance de la fertilité des sols du Burkina Faso. Document Technique $n^{\circ} 1$, Ouagadougou, Burkina Faso, 50p.

Di Vecchia A, Koné B, Djaby B, Labo M, Tarchiani V, De Filippis T, Paganini M, Vignaroli P, Thiombiano L. 2001. Les aptitudes agricoles et pastorales des sols dans les pays du CILSS. Projet Alerte Précoce et Prévision des Productions Agricoles (AP3A) dans le cadre du Programme Majeur Information, 173p.

Dugué P, Vall E, Lecomte P, Klein HD, Rollin D. 2004. Evolution des relations entre l'agriculture et l'élevage dans les savanes d'Afrique de l'Ouest et du
Centre: Un nouveau cadre d'analyse pour améliorer les modes d'intervention et favoriser les processus d'innovation. OCL, 11(4/5): 268-276.

FAO. 2008. Guide de formulation d'une stratégie de mécanisation agricole. Étude de cas: stratégie nationale de la mécanisation agricole au Mali, $65 \mathrm{p}$.

Faure G, Gasselin P, Triomphe B, Temple L, Hocdé H. 2010. Innover avec les Acteurs du Monde Rural, La Recherche-Action en Partenariat. : Editions Quae, Collection Agricultures tropicales en poche: Versailles; $224 \mathrm{p}$.

Guinko S, Fontès J. 1995. Carte de la Végétation et de l'Occupation du Sol du Burkina Faso. Institut de la Carte International de la Végétation. Université Toulouse; Institut du Développement Rural, Université de Ouagadougou: Toulouse, France; Ouagadougou Burkina Faso ; 66 p.

Kribaa M, Hallaire V, Curmi P, Lahmar R. 2001. Effect of different methods of cultivation the structure and hydrolic soil properties in semi arid climate. Soil and Tillage Research, 60: 43-53.

Lahlou S, Ouadia M, Malam Issa O, Le Bissonais Y, Mrabet R. 2005. Modification de la porosité du sol sous les techniques culturales de conservation en zone semi-aride marocaine. Etude et Gestion des sols, 12: 69-76.

Lompo. 2004. Guide pour la Conduite des Evaluations Participatives des Technologies Agricoles. Département GRN/SP, INERA: Burkina Faso; 32p.

Mando A, Ouattara B, Somado AE, Wopereis MCS, Stroosnidjer L, Breman H. 2005. Long term Effects of fallow tillage and manure application on soil organic matter and nitrogen fractions and on sorghum yield under sudano-sahelian conditions. Soil Use and Management, 21: 2531.

Mbetid-Bessane E, Djondang K, Havard M, Kadekoy TD. 2010. Impacts des changements de politique dans un contexte de crise mondialisée sur les acteurs des filières cotonnières d'Afrique 
centrale. Cahiers d'Agriculture, 19(1): 21-27.

Neto MS, Scopel E, Corbeels M, Cardoso AN, Douzet JM, Feller C, Piccolo M, Cerri, CC, Bernoux M. 2010. Soil carbon stocks under no-tillage mulch-based cropping systems in the Brazilian Cerrado: An on-farm synchronic assessment. Soil \& Till Res., 110: 187 195.

Nouiri I, M'hedbi K, Ben Hammouda M, Kammassi M, Neit El Arbi S, Ali Hannachi M, Guesmi L, Mannai C, Hajji S. 2004. Etude comparative de l'humidité des horizons du sol entre le semis direct et le semis conventionnel. Actes des Deuxièmes Rencontres Méditerranéennes sur le Semis Direct.

Pallo FJP, Asimi S, Assa A, Sedogo PM, Sawadogo N. 2006. Statut de la matière organique des sols de la région sahélienne du Burkina Faso. Etude et Gestion des Sols, 13(4): 289-304.

Raubec S. 2001. Le financement de la traction animale en zone de savane cotonnière du Nord-Cameroun dans un contexte de libéralisation. Thèse de doctorat, Montpellier, France, 209 p. + annexes.

Rhyan J, Masri S, Ibrikci H, Singh M, Pala M, Harris HC. 2008. Implication of cereal based crop rotation nitrogen fertilisation and stubble grazing on soil organic matter in a Mediterranean type environment. Turk. J. Agriculture and Forestry, 32: 289-297.
Sangaré M, Poccard Chapuis R, Blanchard M, Bengaly M. 2006. Situation et dynamique agropastorale de Zanférébougou (Mali) : diversité et pratiques. Bobo- Dioulasso (Burkina Faso), CIRDES-IER, Projet Agri-Elevage de Duras.

Sawadogo H, Bock L, Lacroix D, Zombré NP. 2008. Restauration des sols dégradés à l'aide du zaï et du compost dans le Yatenga (Burkina Faso). Biotechnol. Agron. Soc. Environ., 12(3): 279-290.

Son G, Baboui M, Kambiré F, Bourarach E. 2004. Effets du travail du sol sur la levée et le développement racinaire du cotonnier sur sols ferrugineux tropicaux lessivés au Burkina Faso. Revue H.T.E., 129.

Vall E, Dugué P, Blanchard M. 2006. Le tissage des relations agriculture-élevage au fil du coton, 1990-2005. Cahiers Agriculture, 15(1): 72-79.

Walkley A, Black A. 1934. An examination of the Degtjareff method for determining soil organic matter and a proposed modification of the chromic acid titration method. Soil Science, 37: 29-38.

Zougmoré R, Nagumo F, Hosikawa A. 2006. Nutrient uptakes and maize productivity as affected by tillage system and cover crops under the subtropical climate at Ishigaki, Okinawa, Japan. Soil Science \& Plant Nutrition, 52: 509-518. 